\title{
Comparison of the hemolytic activity between $C$. albicans and non-albicans Candida species
}

Rodnei Dennis Rossoni Júnia Oliveira Barbosa Simone Furgeri Godinho Vilela Antonio Olavo Cardoso Jorge Juliana Campos Junqueira

Department of Biosciences and Oral Diagnosis, Institute of Science and Technology, Universidade Estadual Paulista - UNESP, São José dos Campos, SP, Brazil.

Declaration of Interests: The authors certify that they have no commercial or associative interest that represents a conflict of interest in connection with the manuscript.

Corresponding Author:

Rodnei Dennis Rossoni

E-mail: rodnei.rossoni@fosjc.unesp.br

http://dx.doi.org/10.1590/S1806-83242013000600007

Submitted: Dec 18, 2012

Accepted for publication: Jul 31, 2013

Last revision: Aug 14, 2013
Abstract: The ability to produce enzymes, such as hemolysins, is an important virulence factor for the genus Candida. The objective of this study was to compare the hemolytic activity between C. albicans and non-albicans Candida species. Fifty strains of Candida species, isolated from the oral cavity of patients infected with HIV were studied. The isolates included the following species: C. albicans, C. dubliniensis, C. glabrata, C. tropicalis, C. krusei, C. parapsilosis, C. dubliniensis, C. norvegensis, C. lusitaniae, and C. guilliermondii. Hemolysin production was evaluated on Sabouraud dextrose agar containing chloramphenicol, blood, and glucose. A loop-full of pure Candida culture was spot-inoculated onto plates and incubated at $37^{\circ} \mathrm{C}$ for $24 \mathrm{~h}$ in a $5 \% \mathrm{CO}_{2}$ atmosphere. Hemolytic activity was defined as the formation of a translucent halo around the colonies. All C. albicans strains that were studied produced hemolysins. Among the non-albicans Candida species, $86 \%$ exhibited hemolytic activity. Only C. guilliermondii and some C. parapsilosis isolates were negative for this enzyme. In conclusion, most non-albicans Candida species had a similar ability to produce hemolysins when compared to C. albicans.

Descriptors: Candida; Virulence Factors; Acquired Immunodeficiency Syndrome.

\section{Introduction}

The frequency of Candida infection has been gradually increasing over the last several years, accompanied by a significant increase in morbidity and mortality. Candida albicans is the most pathogenic Candida species and is frequently identified in candidiasis lesions in humans. ${ }^{1}$ Twenty years ago, C. albicans represented $80 \%$ of the Candida species recovered from patients with oral and systemic candidiasis. Although $C$. albicans continues to be the most frequently isolated species, the number of infections caused by non-albicans species has increased significantly over the last two decades. ${ }^{2}$

The increased prevalence of non-albicans Candida species found in human candidiasis can be partially attributed to advanced diagnostic methods, such as the use of primary culture media, which are able to differentiate between Candida species, and the introduction of molecular techniques for routine diagnosis. Other factors responsible for the increased prevalence of Candida species include the introduction and 
widespread use of better medical practices (such as immunosuppressive therapy), the administration of broad-spectrum antibiotics, and an increase in the number of invasive surgical procedures. Furthermore, the growing number of Candida species causing candidiasis may be a consequence of species selection in the presence of certain antifungal agents, resulting in the high level of antibiotic resistance found in non-albicans species. ${ }^{2}$

Candida species produce different virulence factors that contribute to colonization, pathogenicity and infection of tissues, including the adhesion to host epithelial cells and biomaterials, the formation of germ tubes and hyphae, the production of hydrolytic enzymes such as proteinases and phospholipases, and hemolytic capacity. ${ }^{3,4}$ Hemolytic capacity is an important virulence factor, that allows fungi of the genus Candida to acquire iron from host tissues, which then is used by the fungus for metabolism, growth and invasion during host infection. ${ }^{5}$

Iron is an essential element for almost all organisms, both unicellular and multicellular. ${ }^{6}$ In humans, iron is found in some proteins, including hemoglobin (a component of erythrocytes). The ability of C. albicans to utilize hemoglobin as an iron source was first described by Moors et al. ${ }^{7}$ According to their study, the first step of C. albicans infection in vivo involves binding to erythrocytes through receptors of the complement system. Next, C. albicans produces a hemolysis factor that induces lysis of the erythrocyte. This factor most likely corresponds to a mannoprotein bound to the cell surface of the fungus. ${ }^{5,8}$ However, the mechanism and molecular basis of hemolysis caused by C. albicans remain unknown. ${ }^{5}$

In the oral cavity, extracellular iron is bound mainly to lactoferrin, a protein present in saliva, while intracellular iron is stored as ferritin. Although this element is bound to proteins and/or is present in the cytoplasm of cells, oral infections with C. albicans are frequent, suggesting that this yeast is able to take up different forms of iron from the oral cavity. ${ }^{9}$ In support of this hypothesis, Almeida et al. ${ }^{9}$ observed that C. albicans caused greater damage to oral epithelial cells containing elevated concentrations of ferritin compared to cells with lower iron levels. In addition, the secretion of hemolysins, followed by the acquisition of iron, facilitates the invasion of hyphae in cases of systemic candidiasis, ${ }^{10}$ and Candida hyphae possess a higher number of hemoglobin receptors than what the yeast form. ${ }^{11}$

Because the number of fungal infections caused by non-albicans Candida species has increased significantly over the last few years and the development of treatment alternatives for fungal infections depends on the study and understanding of virulence factors of these microorganisms, the objective of the present study was to compare the hemolytic capacity of non-albicans Candida species and C. albicans.

\section{Methodology \\ Candida strains}

We studied fifty Candida strains isolated from the oral cavity of HIV-positive patients seen at the Emílio Ribas Institute of Infectious Diseases (Instituto de Infectologia Emílio Ribas - IIER, São Paulo, Brazil). The strains were isolated and identified as previously described by Junqueira et al. ${ }^{12}$ The isolates tested included the following species:

- C. albicans $(\mathrm{n}=20)$,

- C. glabrata $(\mathrm{n}=13)$,

- C. parapsilosis ( $\mathrm{n}=5)$,

- C. dubliniensis $(\mathrm{n}=4)$,

- C. tropicalis $(\mathrm{n}=4)$,

- C. krusei ( $\mathrm{n}=1)$,

- C. guilliermondii $(\mathrm{n}=1)$,

- C. lusitaniae ( $\mathrm{n}=1)$, and

- C. norvegensis $(\mathrm{n}=1)$.

The study was approved by the Ethics Committee of the São José dos Campos Dental School, UNESP (Protocol no. 051/2009/CEP).

All strains were kept in YPD broth (Himedia, Mumbai, India) containing 20\% glycerol (Amresco, Solon, USA) at $-80^{\circ} \mathrm{C}$. The strains were replated on Sabouraud dextrose agar (Himedia, Mumbai, India) and incubated at $37^{\circ} \mathrm{C}$ for $48 \mathrm{~h}$ to analyse hemolysin production.

\section{Hemolysin production}

Hemolysin production was evaluated according 
to methods by Manns et al., ${ }^{13}$ with some modifications. A loop-full of pure Candida culture was inoculated into Sabouraud dextrose agar containing chloramphenicol (Inlab, Diadema, Brazil) and incubated at $37^{\circ} \mathrm{C}$ for $24 \mathrm{~h}$. This growth was used to prepare a suspension of $10^{8}$ cells $/ \mathrm{mL}$ in sterile phosphatebuffered (0.1 M, pH 7.2) saline (Laborclin, Pinhais, Brazil) using a spectrophotometer (B582, Micronal, São Paulo, Brazil). An aliquot $(10 \mu \mathrm{L})$ of the standardized suspension was seeded on to blood agar enriched with glucose (Vetec, Duque de Caxias, Brazil). This medium was prepared with $7 \mathrm{~mL}$ fresh blood per $100 \mathrm{~mL}$ Sabouraud dextrose agar supplemented with chloramphenicol and 3\% glucose. The final $\mathrm{pH}$ was adjusted to $5.6 \pm 0.2$. Plates were incubated at $37^{\circ} \mathrm{C}$ for $48 \mathrm{~h}$ in a $5 \% \mathrm{CO}_{2}$ atmosphere.

Hemolytic activity was measured using the method described by Price et al.,${ }^{14}$ where Pz corresponds to the ratio of the diameter of the colony alone to the diameter of the colony plus the precipitation zone, this is obtained by dividing the colony diameter in $\mathrm{mm}$ by the diameter of the colony plus the halo formed due to enzymatic activity. According to this system, $\mathrm{Pz}=1.00$ indicates that no halo was produced, i.e., there was a lack of enzymatic activity. The lower the value of $\mathrm{Pz}$, the higher the enzymatic activity of the strain. The results were converted into scores (Table 1).

\section{Statistical analysis}

The scores attributed to $\mathrm{Pz}$ values were statistically analyzed by the Mann-Whitney test, using GraphPad Prism 6.0 (GraphPad Software Inc., La Jolla, USA). A level of significance of $5 \%$ was used.

\section{Results}

The results of the hemolytic activity assays obtained for all Candida species are shown in Table 2. Forty-six (92\%) of the 50 Candida strains tested produced hemolysin, with 29 (58\%) exhibiting strong hemolytic activity. C. guilliermondii and three C. parapsilosis strains were the only isolates that did not produce hemolysin.

All C. albicans species (100\%) produced a hemolysis halo, while $86 \%$ of the non-albicans species exhibited hemolysis (Figure 1). Statistical analysis
Table 1 - Enzymatic activity according to Pz value and score attributed (Price et al., ${ }^{14}$ with modifications).

\begin{tabular}{c|c|c}
\hline$P_{z}$ & Enzymatic activity & Score \\
\hline 1.00 & Negative & 0 \\
\hline$\geq 0.64<1.00$ & Positive & 1 \\
\hline$<0.64$ & Strongly positive & 2 \\
\hline
\end{tabular}

Table 2 - Distribution of Candida species according to hemolytic activity score.

\begin{tabular}{c|c|c|c}
\hline \multirow{2}{*}{$\begin{array}{c}\text { Species (number of } \\
\text { isolates) }\end{array}$} & \multicolumn{3}{|c}{ Hemolytic activity score } \\
\cline { 2 - 4 } & Score 2 & Score 1 & Score 0 \\
\hline C. albicans (20) & 10 & 10 & 0 \\
\hline C. glabrata (13) & 12 & 1 & 0 \\
\hline C. parapsilosis (5) & 1 & 1 & 3 \\
\hline C. dubliniensis (4) & 3 & 1 & 0 \\
\hline C. tropicalis (4) & 1 & 3 & 0 \\
\hline C. krusei (1) & 0 & 1 & 0 \\
\hline C. guilliermondii (1) & 0 & 0 & 1 \\
\hline C. lusitaniae (1) & 1 & 0 & 0 \\
\hline C. norvegensis (1) & 1 & 0 & 0 \\
\hline
\end{tabular}

Score 0: no hemolytic activity; score 1: positive hemolytic activity; score 2: strongly positive hemolytic activity.

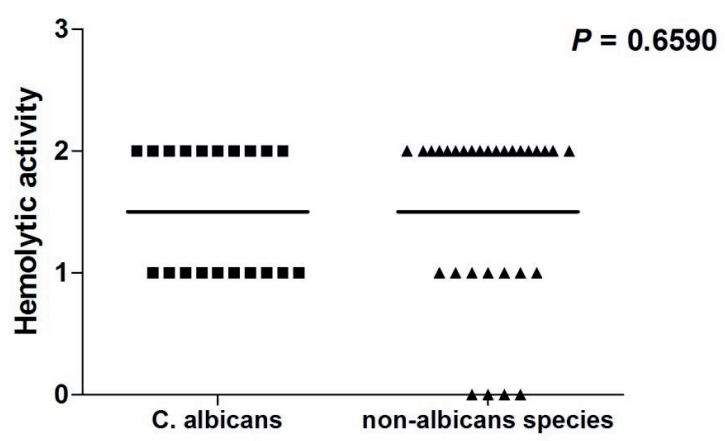

Figure 1 - Values and medians of hemolytic activity observed for C. albicans and non-albicans species ( $p=0.6590)$.

revealed no significant difference between the hemolysis produced by $C$. albicans and non-albicans species $(p=0.6590)$.

As various C. albicans and C. glabrata isolates were analyzed, statistical comparison between these two species was possible. Hemolytic activity was significantly higher in C. glabrata compared to C. 
albicans $(p=0.0140)$. As shown in Table 2, strong positive activity was observed for $50 \%$ of the C. albicans strains and $92 \%$ of the C. glabrata isolates.

\section{Discussion}

In fungi of the genus Candida, the transition from commensalism to pathogenicity can be attributed to the selective expression of different virulence factors that act synergistically under favourable conditions. The type, stage and infection site, in addition to the nature of the immune response, determine which virulence factors the yeast expresses. Among these virulence factors, proteolytic, lipolytic or hemolytic activity seem to play a major role in the pathogenicity of these microorganisms. ${ }^{4,15}$

In the present study, we evaluated hemolysin production, an important virulence factor for yeast from the genus Candida. This enzyme degrades host erythrocytes to release iron for use in growth and metabolism of these fungi in cases of systemic infections. ${ }^{16}$ We also compared the ability of C. albicans and non-albicans Candida species isolated from the oral cavity of HIV-positive patients to produce hemolysin. C. albicans is the predominant species associated with mucosal and systemic fungal infections. However, the epidemiology of yeast infection is rapidly evolving, and non-albicans Candida species have emerged as major opportunistic pathogens primarily in the specific conditions of immunodeficiency, such as acquired immunodeficiency syndrome. ${ }^{12}$

The present results showed that most Candida species $(92 \%)$ produced hemolysins; of these species, 58\% had strongly positive hemolytic activity. These findings agree with those of Ramesh et al. ${ }^{17}$ who compared the hemolytic activity of 50 Candi$d a$ strains isolated from patients with HIV and 10 Candida strains isolated from immunocompetent patients. All strains produced hemolysis, but hemolytic activity was significantly higher for C. albicans strains isolated from HIV patients when compared to those isolated from immunocompetent patients. Mane et al. ${ }^{18}$ also studied the hemolytic ability of Candida isolates from a cohort of 335 patients, composed of $210 \mathrm{HIV}$-positive and 135 HIV-negative individuals. The authors verified that isolates from HIV-positive patients had significantly in- creased production of hemolysin when compared to isolates from HIV-negative individuals. The strongly positive hemolytic activity found in this study (58\%) suggests that Candida strains in HIV-infected individuals have increased expression of virulence attributes and emphasizes the need for further studies on the development of new approaches for therapeutic intervention.

All C. albicans strains analyzed in this study produced a hemolysis halo $(100 \%)$. Similar results have been reported by Tsang et al. ${ }^{19}$ who evaluated the enzymatic activity of proteinase, phospholipase and hemolysin in 126 oral C. albicans isolates. All isolates produced the three enzymes. Shreaz et al. ${ }^{20}$ found $100 \%$ hemolysis in 26 C. albicans strains isolated from the oral cavity. Among the non-albicans species tested, $86 \%$ were positive for hemolysin. As C. albicans is the most virulent species of the genus Candida, ${ }^{21}$ the hemolytic activity of non-albicans species was compared to that of C. albicans and no significant difference was observed, indicating that non-albicans species possess the same hemolytic capacity as C. albicans.

In the present study, $92 \%$ of the C. glabrata isolates were strongly positive for hemolysin, and the hemolytic activity of this species was significantly higher than C. albicans. However, Mane et al. ${ }^{22}$ analyzed the hemolytic production of 65 Candida isolates from HIV-infected individuals and verified that $C$. albicans $(n=39)$ produced more hemolysin compared to all other species, including C. glabrata ( $\mathrm{n}=8$ ). Ramesh et al. ${ }^{17}$ also evaluated the hemolytic capacity of 50 Candida strains from HIV patients, and verified that C. albicans $(n=45)$ produced a significantly higher amount of hemolysin than C. glabrata $(\mathrm{n}=5)$.

The difference between our results and those from previous studies may be due to the number of strains in each species studied. In the present study, we evaluated a number of C. glabrata $(\mathrm{n}=13)$ similar to the number of C. albicans $(\mathrm{n}=20)$ strains, while the number of samples of C. glabrata was much lower compared to C. albicans in previously cited studies. ${ }^{17,22}$ These data show the need for more studies with C. glabrata, which recently was shown to have the ability to produce $\alpha$ or $\beta$ hemolysis., ${ }^{4,23}$ 
This species has emerged as a potential pathogen in the oral cavity of immunocompromised patients and little is known about its role in infection. ${ }^{24}$ In an in vivo study, Jawhara et al. ${ }^{25}$ demonstrated the high pathogenic potential of C. glabrata in a murine model of colitis characterized by weight loss, colon inflammation, and a high mortality rate of the animals.

The only species that did not produce hemolysin in this study were C. guilliermondii and three C. parapsilosis strains. Similar results have been reported in the literature. Seneviratne et al. ${ }^{26}$ analyzed the production of proteinase, phospholipase and hemolysin in 49 bloodstream isolates of Candida obtained from patients in Hong Kong and Finland. In that study, C. albicans, C. glabrata and C. tropicalis exhibited high hemolytic activity, whereas $C$. guilliermondii and C. parapsilosis did not produce this enzyme. Luo et al. ${ }^{23}$ also studied the hemolytic activity of different Candida species and found that only C. parapsilosis did not produce any type of hemolysis.

The present study is the first investigation of hemolytic activity in C. norvegensis, a species responsible for $7 \%$ of candidemia cases. ${ }^{27}$ This fungus was strongly positive for hemolysin, a finding that suggests the capacity of this emerging species to invade and infect an immunocompromised organism.

The four C. tropicalis isolates tested produced hemolysin. França et al. ${ }^{28}$ investigated the hemolytic activity of C. parapsilosis and C. tropicalis isolated from different human anatomical sites. According to their data, enzymatic activity varies widely from

\section{References}

1. Souza RC, Junqueira JC, Rossoni RD, Pereira CA, Munin E, Jorge AO. Comparison of the photodynamic fungicidal efficacy of methylene blue, toluidine blue, malachite green and low-power laser irradiation alone against Candida albicans. Lasers Med Sci. 2010 May;25(3):385-9.

2. Silva S, Henriques M, Hayes A, Oliveira R, Azeredo J, Williams DW. Candida glabrata and Candida albicans co-infection of an in vitro oral epithelium. J Oral Pathol Med. 2011 May;40(5):421-7.

3. Akpan A, Morgan R. Oral candidiasis. Postgrad Med J. 2002 Aug;78(922):455-9. species to species and is associated with the site of isolation. Bloodstream C. tropicalis isolates produced a larger hemolysis halo compared to isolates from the trachea and skin, whereas C. parapsilosis isolated from tracheal secretion exhibited higher enzymatic activity compared to bloodstream isolates. Overall, these results show that yeast of the genus Candida express greater or lesser amounts of hemolysin depending on the anatomical site of isolation and consequent immunological and tissue particularities are required for it to colonize and survive in a host.

The scarcity of studies on the hemolytic activity of emerging Candida species and the absence of differential hemolytic activity between non-albicans Candida species and C. albicans observed in the present study indicate that further investigation is needed to elucidate the exact role of the hemolytic capacity of Candida species in fungal infections.

\section{Conclusion}

Non-albicans Candida species exhibited similar hemolytic capacity as C. albicans. The highest hemolytic activity was observed in C. glabrata, followed by C. albicans. C. guilliermondii and some C. parapsilosis strains were the only isolates that did not produce hemolysins.

\section{Acknowledgements}

This study was supported by the state funding agency Fundação de Amparo à Pesquisa do Estado de São Paulo (FAPESP), Brazil (Grants 2007/544423 and 2012/02184-9).

4. Noumi E, Snoussi M, Hentati H, Mahdouani K, del Castillo L, Valentin E, et al. Adhesive properties and hydrolytic enzymes of oral Candida albicans strains. Mycopathologia. 2010 Apr;169(4):269-78.

5. Almeida RS, Wilson D, Hube B. Candida albicans iron acquisition within the host. FEMS Yeast Res. 2009 Oct;9(7):1000-12.

6. Weinberg ED. Iron availability and infection. Biochim Biophys Acta. 2009 Jul;1790(7):600-5.

7. Moors MA, Stull TL, Blank KJ, Buckley HR, Mosser DM. A role for complement receptor-like molecules in iron acquisition by Candida albicans. J Exp Med. 1992 Jun 1;175(6):1643-51. 
8. Watanabe T, Takano M, Murakami M, Tanaka H, Matsuhisa A, Nakao N, et al. Characterization of a haemolytic factor from Candida albicans. Microbiology. $1999 \mathrm{Mar} ; 145(\mathrm{Pt}$ 3):689-94.

9. Almeida RS, Brunke S, Albrecht A, Thewes S, Laue M, Edwards JE, et al. The hyphal-associated adhesin and invasin Als 3 of Candida albicans mediates iron acquisition from host ferritin. PLoS Pathog. 2008 Nov;4(11):e1000217.

10. Pendrak ML, Roberts DD. Hemoglobin is an effective inducer of hyphal differentiation in Candida albicans. Med Mycol. 2007 Feb;45(1):61-71.

11. Tanaka WT, Nakao N, Mikami T, Matsumoto T. Hemoglobin is utilized by Candida albicans in the hyphal form but not yeast form. Biochem Biophys Res Commun. 1997 Mar 17;232(2):350-3.

12. Junqueira JC, Vilela SF, Rossoni RD, Barbosa JO, Costa AC, Rasteiro VM, et al. Oral colonization by yeasts in HIV-positive patients in Brazil. Rev Inst Med Trop Sao Paulo. 2012 JanFeb;54(1):17-24.

13. Manns JM, Mosser DM, Buckley HR. Production of a hemolytic factor by Candida albicans. Infect Immun. 1994 Nov;62(11):5154-6.

14. Price MF, Wilkinson ID, Gentry LO. Plate method for detection of phospholipase activity in Candida albicans. Sabouraudia. 1982 Mar;20(1):7-14.

15. Mane A, Gaikwad S, Bembalkar S, Risbud A. Increased expression of virulence attributes in oral Candida albicans isolates from human immunodeficiency virus-positive individuals. J Med Microbiol. 2012 Feb;61(Pt 2):285-90.

16. Jeeves RE, Mason RP, Woodacre A, Cashmore AM. Ferric reductase genes involved in high-affinity iron uptake are differentially regulated in yeast and hyphae of Candida albicans. Yeast. 2011 Sep;28(9):629-44.

17. Ramesh N, Priyadharsini M, Sumathi CS, Balasubramanian V, Hemapriya J, Kannan R. Virulence factors and anti fungal sensitivity pattern of Candida sp. isolated from HIV and TB patients. Indian J Microbiol. 2011 Jul;51(3):273-8.

18. Mane A, Gaikwad S, Bembalkar S, Risbud A. Increased expression of virulence attributes in oral Candida albicans isolates from human immunodeficiency virus-positive individuals. J Med Microbiol. 2012 Feb;61(Pt 2):285-90.
19. Tsang CS, Chu FC, Leung WK, Jin LJ, Samaranayake LP, Siu SC. Phospholipase, proteinase and haemolytic activities of Candida albicans isolated from oral cavities of patients with type 2 diabetes mellitus. J Med Microbiol. 2007 Oct;56(Pt 10):1393-8.

20. Shreaz S, Bhatia R, Khan N, Maurya IK, Ahmad SI, Muralidhar S, et al. Cinnamic aldehydes affect hydrolytic enzyme secretion and morphogenesis in oral Candida isolates. Microb Pathog. 2012 May;52(5):251-8.

21. Naglik JR, Moyes DL, Wächtler B, Hube B. Candida albicans interactions with epithelial cells and mucosal immunity. Microbes Infect. 2011 Nov;13(12-13):963-76.

22. Mane A, Pawale C, Gaikwad S, Bembalkar S, Risbud A. Adherence to buccal epithelial cells, enzymatic and hemolytic activities of Candida isolates from HIV-infected individuals. Med Mycol. 2011 Jul;49(5):548-51.

23. Luo G, Samaranayake LP, Yau JY. Candida species exhibit differential in vitro hemolytic activities. J Clin Microbiol. 2001 Aug;39(8):2971-4.

24. Li L, Redding S, Dongari-Bagtzoglou A. Candida glabrata: an emerging oral opportunistic pathogen. J Dent Res. 2007 Mar;86(3):204-15.

25. Jawhara S, Mogensen E, Maggiotto F, Fradin C, Sarazin A, Dubuquoy L, et al. Murine model of dextran sulfate sodiuminduced colitis reveals Candida glabrata virulence and contribution of $\beta$-mannosyltransferases. J Biol Chem. 2012 Mar $30 ; 287(14): 11313-24$.

26. Seneviratne CJ, Wong SS, Yuen KY, Meurman JH, Pärnänen $\mathrm{P}$, Vaara $\mathrm{M}$, et al. Antifungal susceptibility and virulence attributes of bloodstream isolates of Candida from Hong Kong and Finland. Mycopathologia. 2011 Nov;172(5):389-95.

27. Das I, Nightingale P, Patel M, Jumaa P. Epidemiology, clinical characteristics, and outcome of candidemia: experience in a tertiary referral center in the UK. Int J Infect Dis. 2011 Nov;15(11):e759-63.

28. França EJ, Furlaneto-Maia L, Quesada RM, Favero D, Oliveira MT, Furlaneto MC. Haemolytic and proteinase activities in clinical isolates of Candida parapsilosis and Candida tropicalis with reference to the isolation anatomic site. Mycoses. $2011 \mathrm{Jul} ; 54(4): \mathrm{e} 44-51$. 